\title{
Laplace transform of some special functions in terms of gen- eralized Meijer G-functions
}

\author{
Syed Ali Haider Shah*, Shahid Mubeen \\ Department of Mathematics, University of Sargodha, Sargodha, Pakistan.
}

\begin{abstract}
The aim of this paper is to prove Laplace transform of some special functions in term of generalized Meijer G-functions. Some properties of generalized Meijer G-functions will be discussed. We investigate the Laplace transform of different hypergeometric functions in the form of generalized Meijer G-functions and hypergeometric functions. We derive Laplace transform of Bessel k-functions, hyper-Bessel k-functions, incomplete gamma k-function, sine k-integral, sine hyperbolic k-integral, Kelvin $k$-function in the form of generalized Meijer G-functions. In fact, we provide new approach to find Laplace transform of said functions.
\end{abstract}

Keywords: Meijer G-functions, generalized Meijer G-functions, Laplace transform, generalized hypergeometric functions, generalized Bessel functions.

2020 MSC: 33C60, 44A10, 33C20, 33C10.

(C)2022 All rights reserved.

\section{Introduction}

Special functions are applicable in solving variety of problems of mathematical physics, statistics, mechanics, dynamics, functional analysis etc. In recent few decades, many researchers are working on this field. The Fox H-function, Meijer G-function (special case of $\mathrm{H}$-function), Mcrobert E-function (special case of G-function) are particular type of special functions upon which lot of work have been done by the researchers $[1,3,14,17,21,28,30,31,39]$ and found different problems and their solutions associated with these functions in different fields.

The Pochhammer's symbol, gamma function, beta function are the most basic and important functions in the field of special functions.

Definition 1.1. The Pochhammer's symbol can be defined [37] as

$$
(\ell)_{m}= \begin{cases}1, & \text { if } m=0, \ell \in \mathbb{C} \backslash\{0\}, \\ \ell(\ell+1)(\ell+2) \cdots(\ell+m-1), & \text { if } m \in \mathbb{N}, \ell \in \mathbb{C} .\end{cases}
$$

\footnotetext{
*Corresponding author

Email addresses: ali.bukhari78699@gmail.com (Syed Ali Haider Shah), smjhanda@gmail.com (Shahid Mubeen)

doi: $10.22436 /$ jnsa.015.02.03
}

Received: 2021-06-09 Revised: 2021-07-16 Accepted: 2021-07-26 
The relation between Pochhammer symbol and gamma function is given as

$$
(\ell)_{m}=\frac{\Gamma(\ell+m)}{\Gamma(\ell)} \text {. }
$$

Definition 1.2. The Bessel function of first kind is defined by $[4,11]$ as

$$
J_{\vartheta}(l)=\sum_{n=0}^{\infty} \frac{(-1)^{n}}{\Gamma(1+\vartheta+n) n !}\left(\frac{l}{2}\right)^{\vartheta+2 n},
$$

where $\vartheta$ is non-negative integer.

The Mellin-Barnes type contour integrals are the integrals in which gamma functions are used as an integrand. These integrals were first introduced by Pincherle in 1888. In the year 1908, Barnes used the Mellin-Barnes type contour integrals to develop the theory of hypergeometric functions. Meijer Gfunction was introduced by Meijer [18] in the year 1936. On the basis of the theory of Mellin-Barnes type contour integrals, the Meijer G-functions were developed and defined by $[8,14,16,19,20]$ as following.

Definition 1.3.

$$
G_{p, q}^{m, n}\left(\begin{array}{c}
e_{p} \\
f_{q}
\end{array} \mid z\right)=\frac{1}{2 \pi i} \oint_{L} \frac{\prod_{j=1}^{m} \Gamma\left(f_{j}-s\right) \prod_{j=1}^{n}\left(1-e_{j}+s\right)}{\prod_{j=m+1}^{p} \Gamma\left(e_{j}-s\right) \prod_{j=n+1}^{q} \Gamma\left(1-f_{j}+s\right)} z^{s} d s,
$$

where $0 \leqslant \mathrm{~m} \leqslant \mathrm{q}, 0 \leqslant \mathrm{n} \leqslant \mathrm{p}, z \neq 0$, L is the left loop of contour integration starting and ending at $-\infty$ and covering all poles of $\Gamma\left(f_{r}+s\right), r=1,2,3, \ldots, m$ in positive direction but not encircling right series of poles of $\Gamma\left(1-e_{r}-s\right), r=1,2,3, \ldots, n$.

Definition 1.4. The Legendre Duplication formula and Gauss multiplication formula are defined [37] respectively as

$$
\sqrt{\pi} \Gamma(2 \ell)=2^{2 \ell-1} \Gamma(\ell) \Gamma\left(\ell+\frac{1}{2}\right), \quad \Gamma(n \ell)=(2 \pi)^{\frac{1-n}{2}} n^{n \ell-\frac{1}{2}} \Gamma(\ell) \Gamma\left(\ell+\frac{1}{n}\right) \cdots \Gamma\left(\ell+\frac{n-1}{n}\right),
$$

where $n$ is positive integer and $n \ell \in \mathbb{C} \backslash \mathbb{Z}_{0}^{-}$. For $n=3$, we can obtain triplication formula.

Diaz and Pariguan $[5,6]$ gave an idea of k-functions like Pochammer k-symbol, gamma k-function, beta $k$-function and introduced the $k$-form of hypergeometric functions. The researchers [13, 15, 22] worked on this particular k-symbol, developed immense amount of properties and found applications in various fields. In 2017, Nisar et al. [25] introduced ( $k$, s)-fractional integral and differential operators and used them to produce new operators. Nisar et al. [24] introduced k-Struve functions and found different related properties. They also discussed differential equations, integral equations, recurrence relations related to k-Struve function. In 2018, Nisar et al. [26] worked on inequalities by considering k-theory and established Gronwall type inequalities. Rahman et al. [35] gave the extension of the k-Mittag-Leffler function and discussed related properties by using the results proposed by Mubeen et al. [23] for extended gamma and beta functions. Rahman et al. [36] introduced some inequalities involving the k-gamma and $k$-beta functions. They proved the generalization of inequalities for the beta functions.

Definition 1.5. The Pochhammer k-symbol, gamma k-function, and beta $k$-function for $k>0$ are defined [5] respectively as

$$
\begin{aligned}
(\ell)_{m, k} & = \begin{cases}1, & \text { if } m=0, \ell \in \mathbb{C} \backslash\{0\}, \\
\ell(\ell+k)(\ell+2 k) \cdots(\ell+(m-1) k), & \text { if } m \in \mathbb{N}, \ell \in \mathbb{C},\end{cases} \\
\Gamma_{k}(\ell) & =\lim _{m \rightarrow \infty} \frac{m ! k^{m}(m k)^{\frac{\ell}{k}}-1}{(\ell)_{m, k}}, \quad k>0, \zeta \in \mathbb{C} \backslash k Z^{-},
\end{aligned}
$$

and

$$
\beta_{\mathrm{k}}(\ell, \omega)=\frac{1}{\mathrm{k}} \int_{0}^{1} x^{\frac{\ell}{k}-1}(1-x)^{\frac{\omega}{k}-1} \mathrm{~d} x, \quad \mathfrak{R}(\ell)>0, \mathfrak{R}(\omega)>0 .
$$


Definition 1.6. The integral form of gamma k-function is given as

$$
\Gamma_{\mathrm{k}}(\ell)=\int_{0}^{\infty} x^{\frac{\ell}{k}-1} e^{\frac{-x^{k}}{k}} \mathrm{~d} x, \quad \mathfrak{R}(\ell)>0, \mathrm{k}>0 .
$$

Definition 1.7. The Legendre duplication formula and Gauss multiplication formula in k-form are given respectively as

$$
\begin{aligned}
\sqrt{\frac{\pi}{k}} \Gamma(2 \ell) & =2^{\frac{2 \ell}{k}-1} \Gamma(\ell) \Gamma\left(\ell+\frac{k}{2}\right), \\
\Gamma_{k}(n \ell) & =(2 \pi)^{\frac{1-n}{2}} n^{\frac{n \ell}{k}-\frac{1}{2}} k^{\frac{1-n}{2}} \Gamma_{k}(\ell) \Gamma_{k}\left(\ell+\frac{k}{n}\right) \cdots \Gamma_{k}\left(\ell+\frac{(n-1) k}{n}\right),
\end{aligned}
$$

where $n$ is positive integer and $n \ell \in \mathbb{C} \backslash \mathbb{Z}_{0}^{-}$. If we take $k=1$ then we will get the Legendre Duplication formula and Gauss multiplication theorem given in [37, p. 23-24].

Remark 1.8. For $n=3$ in equation (1.2), we will obtain the $k$-form of the Legendre's triplication formula.

Definition 1.9. Euler defined the Laplace transform in 1737 as

$$
\{f(x): p\}=\int_{0}^{\infty} e^{-p x} f(x) d x, \mathfrak{R}(p)>0,
$$

which can be expressed in the form of gamma function as

$$
\int_{0}^{\infty} e^{-p x_{t} t^{\ell-1}} \mathrm{dx}=\frac{\Gamma(\ell)}{\mathrm{p}^{\ell}}
$$

where $\min \{\mathfrak{R}(\ell), \mathfrak{R}(\ell)\}>0$.

The researchers $[7,9,27,29,32,33]$ started to work on the Laplace transformation and wrote a number of books, after the book of Laplace published in 1812. Saif et al. [38] proposed the definition of modified Laplace transform for piecewise defined continuous functions having exponential order. They also proved some important results related to modified Laplace transform. Recently, Agarwal et al. [2] discussed some classical Laplace integral transform for the specific type of functions. They gave generalized Laplace transform known as $\beta$-Laplace integral transform. Mohd [34] worked on the Laplace transformation of hypergeometric functions and proved some important relations in the form of Meijer G-functions.

In our current work, we define the generalized form of Meijer G-function for $k>0$, give some properties of generalized Meijer G-functions, introduce Laplace transform for $k>0$ and prove some results. By using these definitions and results, we investigate the Laplace transform of different hypergeometric functions and some other special functions in the form of generalized Meijer G-functions and generalized hypergeometric functions.

\section{Main results}

In this section, we define generalized Meijer G-function, Laplace transform for $k>0$, give some properties of generalized Meijer G-function and prove some results which play an important role in the theory of special functions and fractional field of analysis.

Definition 2.1. The generalized Meijer G-function for $k>0$ can be defined as

$$
G_{k, p, q}^{m, n}\left[\begin{array}{c}
e_{p} \\
f_{q}
\end{array} \mid z\right]=\frac{1}{2 \pi i} \oint_{L} \frac{\Gamma_{k}\left(f_{1, m}-s\right) \Gamma_{k}\left(k-e_{1, n}+s\right)}{\Gamma_{k}\left(e_{n+1, p}-s\right) \Gamma_{k}\left(k-f_{m+1, q}+s\right)} z^{s / k} d s
$$

where $\Gamma_{k}($.$) is well known gamma k$-function and conditions on $m, n, p, q$ are same as that of Meijer G-function defined in previous section. 
Definition 2.2. Laplace transform for $k>0$ can be defined in the form of gamma $k$-function as

$$
\int_{0}^{\infty} e^{\frac{-p x^{k}}{k}} x^{\frac{\ell}{k}-1} d x=\frac{\Gamma_{k}(\ell)}{p^{\frac{\ell}{k}}}
$$

where $0<\mathfrak{R}(\ell)<1$.

Properties: Some important properties of generalized Meijer G-function are given as follows:

\section{Cancellation}

$$
G_{k, p, q}^{m, n}\left[\begin{array}{c}
e_{1}, e_{2}, \ldots, e_{n}, e_{n+1}, \ldots, e_{p} \\
f_{1}, f_{2}, \ldots, f_{m}, f_{m+1}, \ldots, f_{q-1}, e_{1}
\end{array} \mid z\right]=G_{k, p-1, q-1}^{m, n-1}\left[\begin{array}{c}
e_{2}, e_{3}, \ldots, e_{n}, e_{n+1}, \ldots, e_{p} \\
f_{1}, f_{2}, \ldots, f_{m}, f_{m+1}, \ldots, f_{q-1}
\end{array} \mid z\right]
$$

where $n, p, q>1$.

$$
G_{k, p, q}^{m, n}\left[\begin{array}{c}
e_{1}, e_{2}, \ldots, e_{n}, e_{n+1}, \ldots, e_{p-1}, f_{1} \\
f_{1}, f_{2}, \ldots, f_{m}, f_{m+1}, \ldots, f_{q}
\end{array}\right] z=G_{k, p-1, q-1}^{m-1, n}\left[\begin{array}{c}
e_{1}, e_{2}, e_{3}, \ldots, e_{n}, e_{n+1}, \ldots, e_{p-1} \\
f_{2}, \ldots, f_{m}, f_{m+1}, \ldots, f_{q}
\end{array}\right] z
$$

where $m, p, q>1$.

\section{Translation}

$z^{\alpha} G_{k, p, q}^{m, n}\left[\begin{array}{c}e_{1}, e_{2}, \ldots, e_{n}, e_{n+1}, \ldots, e_{p} \\ f_{1}, f_{2}, \ldots, f_{m}, f_{m+1}, \ldots, f_{q}\end{array} \mid z\right]=G_{k, p, q}^{m, n}\left[\begin{array}{c}e_{1}+\alpha k, e_{2}+\alpha k, \ldots, e_{n}+\alpha k, e_{n+1}+\alpha k, \ldots, e_{p}+\alpha k \\ f_{1}+\alpha k, \ldots, f_{m}+\alpha k, f_{m+1}+\alpha k, \ldots, f_{q}+\alpha k\end{array}\right]$,

\section{Symmetric}

$$
G_{k, p, q}^{m, n}\left[\begin{array}{l}
e_{1}, e_{2}, \ldots, e_{n}, e_{n+1}, \ldots, e_{p} \\
f_{1}, f_{2}, \ldots, f_{m}, f_{m+1}, \ldots, f_{q}
\end{array} \mid z\right]=G_{k, p, q}^{n, m}\left[\begin{array}{c}
k-f_{1}, \ldots, k-f_{m}, k-f_{m+1}, \ldots, k-f_{q} \\
k-e_{1}, k-e_{2}, \ldots, k-e_{n}, k-e_{n+1}, \ldots, k-e_{p} \mid \frac{1}{z}
\end{array}\right] .
$$

If we take $k=1$ in above mentioned properties, then we will obtain the properties discussed in [34].

Now, we prove a theorem related to k-Laplace transform which will then be applied in the next sections to prove Laplace transform of different generalized hypergeometric functions in term of generalized Meijer G-functions.

Theorem 2.3. Let $\mathrm{A} \leqslant \mathrm{B}+1, \mathfrak{R}(\mathrm{p})>0, \mathfrak{R}(\mathrm{g})>0, \mathrm{r}$ is a positive integer, $\mathrm{k}>0,|z|<1$ and $\left(\boldsymbol{e}_{\mathrm{A}}\right),\left(f_{\mathrm{B}}\right)$ be the abbreviation of the parameters $e_{1}, e_{2}, \ldots, e_{A}, f_{1}, f_{2}, \ldots, f_{B}$, respectively, then the Laplace transform of hypergeometric functions in term of generalized Meijer G-functions hold as follows

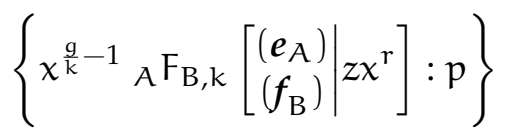

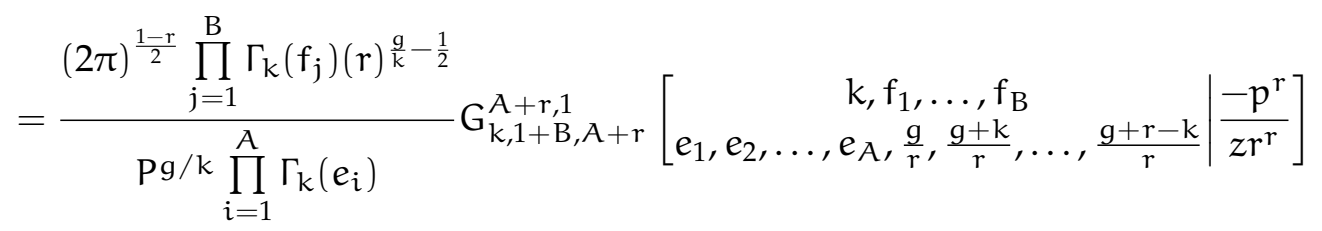

$$
\begin{aligned}
& =\frac{(2 \pi)^{\frac{1-r}{2}} \prod_{j=1}^{B} \Gamma_{k}\left(f_{j}\right)(r)^{\frac{g}{k}-\frac{1}{2}}}{P^{g / k} \prod_{i=1}^{A} \Gamma_{k}\left(e_{i}\right)} \\
& \times G_{k, A+r, 1+B}^{1, A+r}\left[\begin{array}{c}
k-e_{1}, k-e_{2}, \ldots, k-e_{A}, k+\frac{g}{r}, k+\frac{g+k}{r}, \ldots, k+\frac{g+r-k}{r} \mid \frac{-z r^{r}}{p^{r}} \\
0, k-f_{1}, \ldots, k-f_{B}
\end{array}\right] .
\end{aligned}
$$


Proof. Consider the left side of the above equation

$$
\begin{aligned}
\left\{x^{\frac{g}{k}-1}{ }_{A} F_{B, k}\left[\begin{array}{c}
\left(\mathbf{e}_{A}\right) \\
\left(\mathbf{f}_{B}\right)
\end{array} \mid z x^{r}\right]: p\right\} & =\int_{0}^{\infty} e^{\frac{-p x^{k}}{k}} x^{\frac{g}{k}-1}{ }_{A} F_{B, k}\left[\begin{array}{c}
\left(\mathbf{e}_{A}\right) \\
\left(\mathbf{f}_{B}\right)
\end{array} \mid z x^{r}\right] d x \\
& =\int_{0}^{\infty} e^{\frac{-p x^{k}}{k}} x^{\frac{g}{k}-1}\left[\frac{1}{2 \pi \omega} \frac{\prod_{j=1}^{B} \Gamma_{k}\left(f_{j}\right)}{\prod_{i=1}^{A} \Gamma_{k}\left(e_{i}\right)} \int_{l} \frac{\Gamma_{k}(-s) \prod_{i=1}^{A} \Gamma_{k}\left(e_{i}+s\right)\left(-z x^{r}\right)^{\frac{s}{k}}}{\prod_{j=1}^{B} \Gamma_{k}\left(f_{j}+s\right)} d s\right] d x,
\end{aligned}
$$

where $l$ is Mellin-Barnes type contour. So,

$$
\begin{aligned}
& \left\{x^{\frac{g}{k}-1}{ }_{A} F_{B, k}\left[\begin{array}{c}
\left(\mathbf{e}_{A}\right) \\
\left(f_{B}\right)
\end{array} \mid z x^{r}\right]: p\right\} \\
& =\frac{1}{2 \pi \omega} \frac{\prod_{j=1}^{B} \Gamma_{k}\left(f_{j}\right)}{\prod_{i=1}^{A} \Gamma_{k}\left(e_{i}\right)} \int_{l} \frac{\Gamma_{k}(-s) \prod_{i=1}^{A} \Gamma_{k}\left(e_{i}+s\right)(-z)^{\frac{s}{k}}}{\prod_{j=1}^{B} \Gamma_{k}\left(f_{j}+s\right)}\left(\int_{0}^{\infty} e^{\frac{-p x^{k}}{k}} x^{\frac{g+r s}{k}-1} d x\right) d s \\
& =\frac{1}{p^{\frac{g}{k}} 2 \pi \omega} \frac{\prod_{j=1}^{B} \Gamma_{k}\left(f_{j}\right)}{\prod_{i=1}^{A} \Gamma_{k}\left(e_{i}\right)} \int_{l} \frac{\Gamma_{k}(-s) \prod_{i=1}^{A} \Gamma_{k}\left(e_{i}+s\right) \Gamma_{k}(g+r s)\left(\frac{-z}{p^{r}}\right)^{\frac{s}{k}}}{\prod_{j=1}^{B} \Gamma_{k}\left(f_{j}+s\right)} d s \\
& =\frac{(2 \pi)^{\frac{1-r}{2}} r^{\frac{g}{k}-\frac{1}{2}}}{p^{\frac{g}{k}} 2 \pi \omega} \frac{\prod_{j=1}^{B} \Gamma_{k}\left(f_{j}\right)}{\prod_{i=1}^{A} \Gamma_{k}\left(e_{i}\right)} \int_{l} \frac{\Gamma_{k}(-s) \prod_{i=1}^{A} \Gamma_{k}\left(e_{i}+s\right) \prod_{n=1}^{r} \Gamma_{k}\left(s+\frac{g+n-k}{r}\right)\left(\frac{-z r^{r}}{p^{r}}\right)^{\frac{s}{k}}}{\prod_{j=1}^{B} \Gamma_{k}\left(f_{j}+s\right)} d s \\
& =\frac{(2 \pi)^{\frac{1-r}{2}} r^{\frac{g}{k}-\frac{1}{2}}}{p^{\frac{g}{k}}} \frac{\prod_{j=1}^{B} \Gamma_{k}\left(f_{j}\right)}{\prod_{i=1}^{A} \Gamma_{k}\left(e_{i}\right)} G_{k, A+r, 1+B}^{1, A+r}\left[\begin{array}{c}
k-\mathbf{e}_{A}, k-\frac{g+r-k}{r} \\
0, k-\mathbf{f}_{B}
\end{array} \mid \frac{-z r^{r}}{p^{r}}\right]
\end{aligned}
$$

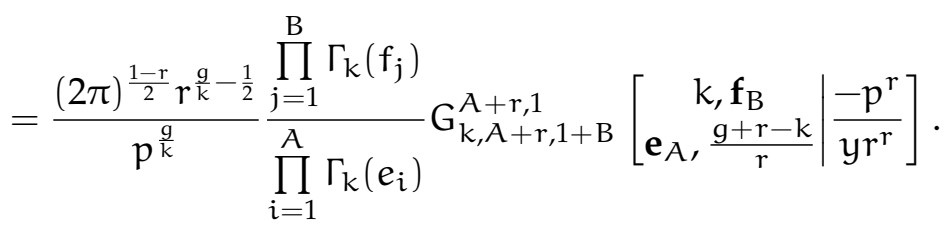

Similarly, we can prove (2.2), by applying formula (1.2), properties of generalized Meijer G-function and definition of Laplace transform for $k>0$.

Now, we prove theorems which give the relations of Bessel functions with hypergeometric k-functions. These theorems will be helpful in the last section of this work to prove the Laplace transform of some known Bessel functions in term of generalized Meijer G-functions.

Theorem 2.4. Let $\vartheta$ be a non-negative integer, $\mathfrak{R}(y)>0, k>0$ and $|y|<1$, then the following holds true:

$$
J_{\vartheta, k}(y) J_{-\vartheta, k}(y)=\frac{\sin \left(\frac{\pi \vartheta}{k}\right)}{\pi \vartheta}{ }_{1} F_{2, k}\left[\begin{array}{c}
\frac{k}{2} \\
k+\vartheta, k-\vartheta
\end{array} \mid-y^{2}\right] .
$$


Proof. Consider the L.H.S

$$
\begin{aligned}
J_{\vartheta, k}(y) J_{-\vartheta, k}(y) & =\sum_{n=0}^{\infty} \frac{(-1)^{n} y^{2 n+\frac{\vartheta}{k}}}{2^{2 n+\frac{\vartheta}{k} n ! \Gamma_{k}(k+n k+\vartheta)}} \sum_{m=0}^{\infty} \frac{(-1)^{m} y^{2 m-\frac{\vartheta}{k}}}{2^{2 m-\frac{\vartheta}{k} m ! \Gamma_{k}(k+m k-\vartheta)}} \\
& =\sum_{n=0}^{\infty} \sum_{m=0}^{\infty} \frac{(-1)^{n+m} y^{2 n+2 m}}{2^{2 n+2 m} n ! m ! \Gamma_{k}(k+n k+\vartheta) \Gamma_{k}(k+m k-\vartheta)}
\end{aligned}
$$

After some simplifications we obtain:

$$
\begin{aligned}
J_{\vartheta, k}(y) J_{-\vartheta, k}(y) & =\frac{1}{\Gamma_{k}(k+\vartheta) \Gamma_{k}(k-\vartheta)} \sum_{n=0}^{\infty} \frac{(-1)^{n} y^{2 n}}{2^{2 n} n !(k+\vartheta)_{n, k}} F_{1, k}\left[\begin{array}{c}
(-n, 1),(-\vartheta-n k) \\
(k-\vartheta, k)
\end{array} \mid 1\right] \\
& =\frac{1}{\Gamma_{k}(k+\vartheta) \Gamma_{k}(k-\vartheta)} \sum_{n=0}^{\infty} \frac{(-1)^{n} y^{2 n}}{2^{2 n} n !} \frac{\Gamma_{k}(k-\vartheta) \Gamma_{k}(k+2 n k)}{\Gamma_{k}(k-\vartheta+n k) \Gamma_{k}(k+n k)} \\
& =\frac{1}{\vartheta \Gamma_{k}(\vartheta) \Gamma_{k}(k-\vartheta)} \sum_{n=0}^{\infty} \frac{(-1)^{n}\left(\frac{k}{2}\right)_{n, k} y^{2 n}}{(k+\vartheta)_{n, k}(k-\vartheta)_{n, k} n !} \\
& =\frac{\sin \left(\frac{\pi \vartheta}{k}\right)}{\pi \vartheta}{ }_{1} F_{2, k}\left[\begin{array}{c}
\frac{k}{2} \\
k+\vartheta
\end{array}-\vartheta \mid-y^{2}\right] .
\end{aligned}
$$

Theorem 2.5. Let $\vartheta, \mu$ be non-negative integers, $\mathfrak{R}(\mathrm{y})>0, \mathrm{k}>0$, then for $|\mathrm{y}|<1$, the following holds true

$$
J_{\mu, k}(y) J_{\vartheta, k}(y)=\frac{\left(\frac{y}{2}\right)^{\frac{\mu+\vartheta}{k}}}{\Gamma_{k}(\mu+k) \Gamma_{k}(\vartheta+k)}{ }_{3} F_{2, k}\left[\begin{array}{c}
\frac{\mu+\vartheta+k}{2}, \frac{\mu+\vartheta+2 k}{2} \\
\mu+k, \vartheta+k, k+\mu+\vartheta
\end{array} \mid-y^{2}\right] .
$$

Proof.

$$
\begin{aligned}
J_{\mu, k}(y) J_{\vartheta, k}(y) & =\sum_{n=0}^{\infty} \frac{(-1)^{n} y^{2 n+\frac{\mu}{k}}}{2^{2 n+\frac{\mu}{k} n ! \Gamma_{k}(k+n k+\mu)}} \sum_{m=0}^{\infty} \frac{(-1)^{m} y^{2 m+\frac{\vartheta}{k}}}{2^{2 m+\frac{\vartheta}{k} m ! \Gamma_{k}(k+m k+\vartheta)}} \\
& =\sum_{n=0}^{\infty} \sum_{m=0}^{\infty} \frac{(-1)^{n+m} y^{2 n+2 m+\frac{\mu}{k}+\frac{\vartheta}{k}}}{2^{2 n+2 m+\frac{\mu}{k}+\frac{\vartheta}{k}} n ! m ! \Gamma_{k}(k+n k+\mu) \Gamma_{k}(k+m k+\vartheta)}
\end{aligned}
$$

After some simplifications we obtain:

$$
\begin{aligned}
J_{\mu, k}(y) J_{\vartheta, k}(y) & \left.=\frac{\left(\frac{y}{2}\right)^{\frac{\mu+\vartheta}{k}}}{\Gamma_{k}(k+\mu) \Gamma_{k}(k+\vartheta)} \sum_{n=0}^{\infty} \frac{(-1)^{n} y^{2 n}}{2^{2 n} n !(k+\mu)_{n, k}}{ }_{2} F_{1, k}\left[\begin{array}{c}
(-n, 1),(-\mu-n k) \mid \\
(k+\vartheta, k)
\end{array}\right] 1\right] \\
& =\frac{\left(\frac{y}{2}\right)^{\frac{\mu+\vartheta}{k}}}{\Gamma_{k}(k+\mu) \Gamma_{k}(k+\vartheta)} \sum_{n=0}^{\infty} \frac{(-1)^{n} y^{2 n}}{2^{2 n}(\mu+k)_{n, k} n !} \frac{\Gamma_{k}(k+\vartheta) \Gamma_{k}(k+2 n k+\mu+\vartheta)}{\Gamma_{k}(k+\vartheta+n k) \Gamma_{k}(k+n k+\mu+\vartheta)} . \\
& =\frac{\left(\frac{y}{2}\right)^{\frac{\mu+\vartheta}{k}}}{\Gamma_{k}(\mu+k) \Gamma_{k}(\vartheta+k)}{ }_{3} F_{2, k}\left[\begin{array}{c}
\frac{\mu+\vartheta+k}{2}, \frac{\mu+\vartheta+2 k}{2} \\
\mu+k, \vartheta+k, k+\mu+\vartheta
\end{array} \mid-y^{2}\right] .
\end{aligned}
$$

Remark 2.6. By adopting the same procedure as that of Theorems 2.4 and 2.5, we can prove the product of Bessel functions with different combination of parameters that are $J_{\vartheta, k}(y) J_{\vartheta+1, k}(y), J_{\vartheta, k}^{2}(y), J_{\vartheta, k}(y) I_{\vartheta, k}(y)$ and hyper-Bessel function $\mathrm{J}_{l, h, k}(\mathrm{y})$, respectively in the form of hypergeometric $k$-functions. 


\section{Laplace transform of some special functions}

In this section, we investigate Laplace transform of different hypergeometric functions in terms of generalized Meijer G-functions for multiple choices of parameters in (2.1) by using some known results of special functions, i.e., Legendre's duplication and triplication formulas (1.1) and (1.2) for the product of gamma k-functions, cancellation properties and reduction formulas etc.

Case-1: By choosing $g=k, A=3, B=2, e_{1}=e, e_{2}=f, e_{3}=g, f_{1}=k+e-f, f_{2}=k+e-g, z=1, r=1$ in equation (2.1), we have

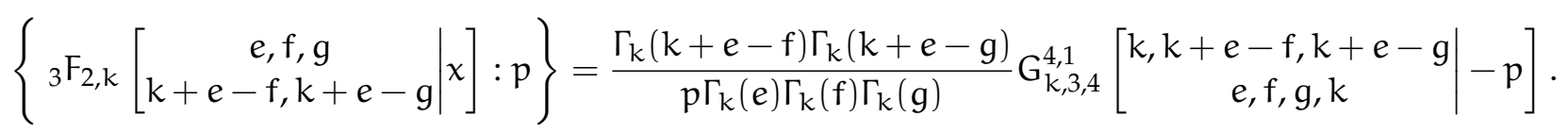

By using Whipple's theorem (see, pg.90, [37]) in k-form, we obtain:

$$
\begin{aligned}
& \left\{(1-k x)^{\frac{-e}{k}}{ }_{3} F_{2, k}\left[\begin{array}{c}
\frac{e}{k}, \frac{f+k}{2}, k+e-f-g \\
k+e-f, k+e-g
\end{array} \mid \frac{-4 x}{(1-k x)^{2}}\right]: p\right\} \\
& =\frac{\Gamma_{k}(k+e-f) \Gamma_{k}(k+e-g)}{p \Gamma_{k}(e) \Gamma_{k}(f) \Gamma_{k}(g)} G_{k, 3,4}^{4,1}\left[\begin{array}{c}
k, k+e-f, k+e-g \\
e, f, g, k
\end{array} \mid-p\right],
\end{aligned}
$$

where $k+e-f, K+e-g, e, f, g \in \mathbb{C} \backslash \mathbb{Z}_{0}^{-}, k>0$.

Case-2: By choosing $g=k, A=3, B=2, e_{1}=e, e_{2}=2 f-e-k, e_{3}=e+2 k-2 f, f_{1}=f, f_{2}=e-f+\frac{3 k}{2}$, $z=\frac{1}{4}, \mathrm{r}=1$ in $(2.1)$, we have

$$
\begin{aligned}
& \left\{{ }_{3} F_{2, k}\left[\begin{array}{c}
e, 2 f-e-k, e-2 f+2 k \\
f, e-f+\frac{3 k}{2}
\end{array} \mid x\right]: p\right\} \\
& =\frac{\Gamma_{k}(f) \Gamma_{k}\left(e-f+\frac{3 k}{2}\right)}{p \Gamma_{k}(e) \Gamma_{k}(2 f-e-k) \Gamma_{k}(e+2 k-2 f)} G_{k, 3,4}^{4,1}\left[\begin{array}{c}
k, f, e-f+\frac{3 k}{2} \\
e, 2 f-e-k, e-2 f+2 k, k
\end{array} \mid-4 p\right] .
\end{aligned}
$$

By using k-form of Whipple's theorem, we find

$$
\begin{aligned}
& \left\{(1-k x)^{\frac{-e}{k}}{ }_{3} F_{2, k}\left[\begin{array}{l}
\frac{e}{3}, \frac{e+k}{3}, \frac{e+2 k}{3} \\
f, e-f+\frac{3 k}{2}
\end{array} \mid \frac{-27 x}{4(1-k x)^{3}}\right]: p\right\} \\
& =\frac{\Gamma_{k}(f) \Gamma_{k}\left(e-f+\frac{3 k}{2}\right)}{p \Gamma_{k}(e) \Gamma_{k}(2 f-e-k) \Gamma_{k}(e-2 f+2 k)} G_{k, 3,4}^{4,1}\left[\begin{array}{c}
k, f, e-f+\frac{3 k}{2} \\
e, 2 f-e-k, e+2 k-2 f, k
\end{array} \mid-4 p\right] \text {, }
\end{aligned}
$$

where $e, f, e-f+\frac{3 k}{2}, 2 f-e-k, e-2 f+2 k \in \mathbb{C} \backslash \mathbb{Z}_{0}^{-}, k>0$.

Case-3: By choosing $g=k, A=3, B=2, e_{1}=e, e_{2}=f-\frac{k}{2}, e_{3}=k+e-f, f_{1}=2 f, f_{2}=2 e-2 f+2 k$, $z=1, r=1$ in (2.1) and using whipple's $k$-theorem, we have

$$
\begin{aligned}
& \left\{\left(1-\frac{k x}{4}\right)^{\frac{-e}{k}}{ }_{3} F_{2, k}\left[\begin{array}{l}
\frac{e}{3}, \frac{e+k}{3}, \frac{e+2 k}{3} \\
f, e-f+\frac{3 k}{2}
\end{array} \mid \frac{-27 k x^{2}}{(4-k x)^{3}}\right]: p\right\} \\
& =\frac{2^{\frac{2 e}{k}} k\left(f-\frac{k}{2}\right) \Gamma_{k}(f) \Gamma_{k}\left(e-f+\frac{3 k}{2}\right)}{\pi p \Gamma_{k}(e)} G_{k, 3,4}^{4,1}\left[\begin{array}{c}
k, 2 f, 2 e-2 f+2 k \\
e, f-\frac{k}{2}, e+k-f, k
\end{array} \mid-p\right],
\end{aligned}
$$

where $e, f, e-f+\frac{3 k}{2} \in \mathbb{C} \backslash \mathbb{Z}_{0}^{-}, k>0$.

Case-4: By choosing $g=k, A=2, B=1, e_{1}=e, e_{2}=f, f_{1}=2 e, z=1, r=1$ in (2.1), we have

$$
\left\{{ }_{2} F_{1, k}\left[\begin{array}{c}
e, f \\
2 e
\end{array} \mid x\right]: p\right\}=\frac{\Gamma_{k}(2 e)}{p \Gamma_{k}(e) \Gamma_{k}(f)} G_{k, 2,3}^{3,1}\left[\begin{array}{c}
k, 2 e \\
e, f, k
\end{array} \mid-p\right] \text {. }
$$


By using k-form of (see, pg.65, [37]), we obtain

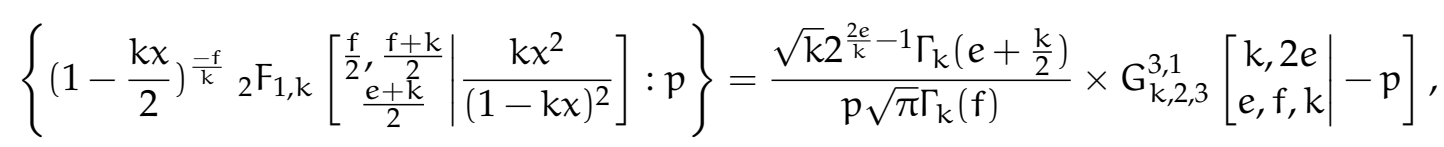

where $e+\frac{k}{2}, f \in \mathbb{C} \backslash \mathbb{Z}_{0}^{-}, k>0$.

Case-5: By choosing $g=k, A=2, B=1, e_{1}=e, e_{2}=e+\frac{k}{2}-f, f_{1}=f+\frac{k}{2}, z=1, r=2$ in (2.1), we have

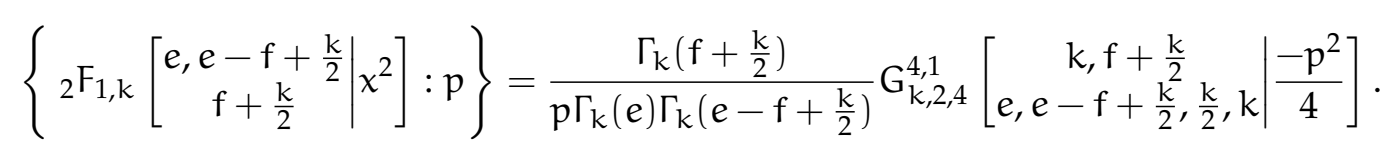

By using the result

$$
(1+k x)^{\frac{2 e}{k}}{ }_{2} F_{1, k}\left[\begin{array}{c}
e, e-f+\frac{k}{2} \\
f+\frac{k}{2}
\end{array} \mid x^{2}\right]={ }_{2} F_{1, k}\left[\begin{array}{c|c}
e, f & 4 x \\
2 e & \frac{4 x}{(1+k x)^{2}}
\end{array}\right]
$$

we have

$$
\left\{(1+k x)^{\frac{-2 e}{k}}{ }_{2} F_{1, k}\left[\begin{array}{c}
e, f \\
2 f
\end{array} \mid \frac{4 x}{(1+k x)^{2}}\right]: p\right\}=\frac{\Gamma_{k}\left(f+\frac{k}{2}\right)}{p \Gamma_{k}(e) \Gamma_{k}\left(e-f+\frac{k}{2}\right)} G_{k, 2,4}^{4,1}\left[\begin{array}{c}
k, f+\frac{k}{2} \\
e, e-f+\frac{k}{2}
\end{array} \mid \frac{-p^{2}}{4}\right],
$$

where $e-f+\frac{k}{2}, e, f+\frac{k}{2} \in \mathbb{C} \backslash \mathbb{Z}_{0}^{-}, k>0$.

Case-6: By choosing $g=k, A=2, B=1, e_{1}=2 e, e_{2}=2 f, f_{1}=e+f+\frac{k}{2}, z=1, r=1$ in (2.1), we have

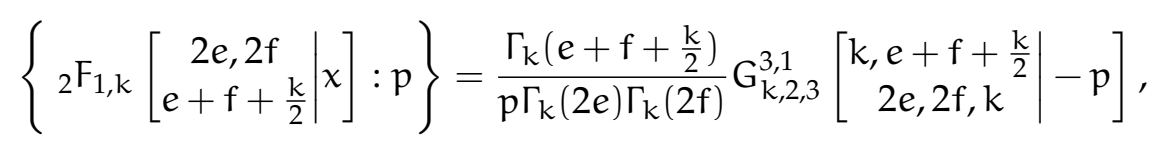

$$
\begin{aligned}
& \left\{{ }_{2} F_{1, k}\left[\begin{array}{c}
e, f \\
e+f+\frac{k}{2}
\end{array} \mid 4 x(1-k x)\right]: p\right\}=\frac{\Gamma_{k}\left(e+f+\frac{k}{2}\right)}{p \Gamma_{k}(e) \Gamma_{k}(f)} G_{k, 2,3}^{3,1}\left[\begin{array}{c}
k, e+f+\frac{k}{2} \\
2 e, 2 f, k
\end{array} \mid-p\right],
\end{aligned}
$$

where $e+f+\frac{k}{2}, 2 e, 2 f \in \mathbb{C} \backslash \mathbb{Z}_{0}^{-}, k>0$.

Case-7: By choosing $g=k, A=3, B=2, e_{1}=2 e, e_{2}=2 f, e_{3}=e+f, f_{1}=2 e+2 f-k, f_{2}=e+f+\frac{k}{2}$, $z=1, r=1$, in (2.1), we have

$$
\left\{{ }_{3} F_{2, k}\left[\begin{array}{c}
2 e, 2 f, e+f \\
2 e+2 f-k, e+f+\frac{k}{2} \mid
\end{array} \mid x\right]: p\right\}=\frac{\Gamma_{k}(2 e+2 f-k) \Gamma_{k}\left(e+f+\frac{k}{2}\right)}{p \Gamma_{k}(2 e) \Gamma_{k}(2 f) \Gamma_{k}(e+f)} G_{k, 3,4}^{4,1}\left[\begin{array}{c}
k, 2 e+2 f-k, e+f+\frac{k}{2} \\
2 e, 2 f, e+f, k
\end{array} \mid-p\right] .
$$

After using Legendre's Duplication formula in k-form (1) and little simplification, we are at the following result:

$$
\begin{aligned}
& \left\{{ }_{2} F_{1, k}\left[\begin{array}{c}
e, f \\
e+f-\frac{k}{2} \mid x
\end{array} \mid{ }_{2} F_{1, k}\left[\begin{array}{c}
e, f \\
e+f+\frac{k}{2} \mid x
\end{array}\right]: p\right\}=\frac{\sqrt{\pi} \Gamma_{k}\left(e+f-\frac{k}{2}\right) \Gamma_{k}\left(e+f+\frac{k}{2}\right)}{\sqrt{k} p \Gamma_{k}(e) \Gamma_{k}\left(e+\frac{k}{2}\right) \Gamma_{k}(f) \Gamma_{k}\left(f+\frac{k}{2}\right)}\right. \\
& \times G_{k, 3,4}^{4,1}\left[\begin{array}{c}
k, 2 e+2 f-k, e+f+\frac{k}{2} \\
2 e, 2 f, e+f, k
\end{array} \mid-p\right],
\end{aligned}
$$

where $e+f+\frac{k}{2}, e+f-\frac{k}{2}, e, f, e+\frac{k}{2}, f+\frac{k}{2} \in \mathbb{C} \backslash \mathbb{Z}_{0}^{-}, k>0$.

Case-8: By choosing $g=k, A=3, B=2, e_{1}=2 e, e_{2}=2 f-k, e_{3}=e+f-k, f_{1}=2 e+2 f-2 k$, $f_{2}=e+f-\frac{k}{2}, z=1, r=1$ in (2.1), we have

$$
\left\{{ }_{3} F_{2, k}\left[\begin{array}{c}
2 e, 2 f-k, e+f-k \\
2 e+2 f-2 k, e+f-\frac{k}{2}
\end{array} \mid x\right]: p\right\}=\frac{\Gamma_{k}(2 e+2 f-2 k) \Gamma_{k}\left(e+f-\frac{k}{2}\right)}{p \Gamma_{k}(2 e) \Gamma_{k}(2 f-k) \Gamma_{k}(e+f-k)} G_{k, 3,4}^{4,1}\left[\begin{array}{c}
k, 2 e+2 f-k, e+f-\frac{k}{2} \\
2 e, 2 f-k, e+f-k, k
\end{array} \mid-p\right] .
$$


After using (1.1) and little simplification, we obtain:

$$
\begin{aligned}
& \left\{{ }_{2} F_{1, k}\left[\begin{array}{c}
e, f \\
e+f-\frac{k}{2} \mid x
\end{array}\right]{ }_{2} F_{1, k}\left[\begin{array}{c}
e, f-k \\
e+f-\frac{k}{2} \mid x
\end{array}\right]: p\right\}=\frac{\sqrt{\pi} \Gamma_{k}\left(e+f-\frac{k}{2}\right) \Gamma_{k}\left(e+f-\frac{k}{2}\right)}{\sqrt{k} p \Gamma_{k}(e) \Gamma_{k}\left(e+\frac{k}{2}\right) \Gamma_{k}(f) \Gamma_{k}\left(f-\frac{k}{2}\right)} \\
& \times G_{k, 3,4}^{4,1}\left[\begin{array}{c}
k, 2 e+2 f-k, e+f-\frac{k}{2} \\
2 e, 2 f-k, e+f-k, k
\end{array} \mid-p\right] \text {, }
\end{aligned}
$$

where $e+f-\frac{k}{2}, e, f, e+\frac{k}{2}, f-\frac{k}{2} \in \mathbb{C} \backslash \mathbb{Z}_{0}^{-}, k>0$.

Case-9: By choosing $g=k, A=3, B=2, e_{1}=e-f+\frac{k}{2}, e_{2}=f-e+\frac{k}{2}, e_{3}=\frac{k}{2}, f_{1}=e+f+\frac{k}{2}$, $f_{2}=\frac{3 k}{2}-e-f, z=1, r=1$ in (2.1), we have

$$
\begin{aligned}
\left\{{ }_{3} F_{2, k}\left[\begin{array}{l}
e-f+\frac{k}{2}, f-e+\frac{k}{2}, \frac{k}{2} \\
e+f+\frac{k}{2},+\frac{3 k}{2}-e-f
\end{array} \mid: p\right\}=\right. & \frac{\Gamma_{k}\left(e+f+\frac{k}{2}\right) \Gamma_{k}\left(\frac{3 k}{2}-e-f\right)}{p \Gamma_{k}\left(e-f+\frac{k}{2}\right) \Gamma_{k}\left(f-e+\frac{k}{2}\right) \Gamma_{k}\left(\frac{k}{2}\right)} \\
& \times G_{k, 3,4}^{4,1}\left[\begin{array}{c}
k, e+f+\frac{k}{2}, \frac{3 k}{2}-e-f \\
e-f+\frac{k}{2}, f-e+\frac{k}{2}, \frac{k}{2}, k
\end{array} \mid-p\right] .
\end{aligned}
$$

After some simplifications we get the following:

$$
\begin{aligned}
\left\{{ }_{2} F_{1, k}\left[\begin{array}{c}
e, f \\
e+f+\frac{k}{2} \mid x
\end{array}\right]{ }_{2} F_{1, k}\left[\begin{array}{c}
\frac{k}{2}-e, \frac{k}{2}-f \\
\frac{3 k}{2}-e-f
\end{array}\right]: p\right\}= & \frac{\sqrt{k} \Gamma_{k}\left(e+f+\frac{k}{2}\right) \Gamma_{k}\left(\frac{3 k}{2}-e-f\right)}{\sqrt{\pi} p \Gamma_{k}\left(e-f+\frac{k}{2}\right) \Gamma_{k}\left(f-e+\frac{k}{2}\right)} \\
& \times G_{k, 3,4}^{4,1}\left[\begin{array}{c}
k, e+f+\frac{k}{2}, \frac{3 k}{2}-e-f \\
e-f+\frac{k}{2}, f-e+\frac{k}{2}, \frac{k}{2}, k
\end{array} \mid-p\right],
\end{aligned}
$$

where $e+f+\frac{k}{2}, \frac{3 k}{2}-e-f, f-e+\frac{k}{2}, e-f+\frac{k}{2} \in \mathbb{C} \backslash \mathbb{Z}_{0}^{-}, k>0$.

Case-10: By choosing $g=k, A=3, B=2, e_{1}=2 e, e_{2}=2 f, e_{3}=e+f, f_{1}=2 e+2 f, f_{2}=e+f+\frac{k}{2}, z=1$, $r=1$ in (2.1), we have

$$
\left\{{ }_{3} F_{2, k}\left[\begin{array}{c}
2 e, 2 f, e+f \\
2 e+2 f, e+f+\frac{k}{2}
\end{array} \mid x\right]: p\right\}=\frac{\Gamma_{k}(2 e+2 f) \Gamma_{k}\left(\frac{k}{2}+e+f\right)}{p \Gamma_{k}(2 e) \Gamma_{k}(2 f) \Gamma_{k}(e+f)} G_{k, 3,4}^{4,1}\left[\begin{array}{c}
k, 2 e+2 f, \frac{k}{2}+e+f \\
2 e, 2 f, e+f, k
\end{array} \mid-p\right] .
$$

After some simplifications, we get the following:

$$
\begin{aligned}
\left\{\left({ }_{2} F_{1, k}\left[\begin{array}{c}
e, f \\
e+f+\frac{k}{2}
\end{array} \mid x\right]\right)^{2}: p\right\}= & \frac{\sqrt{\pi} \Gamma_{k}\left(e+f+\frac{k}{2}\right) \Gamma_{k}\left(\frac{k}{2}+e+f\right)}{\sqrt{k} 2 p \Gamma_{k}(e) \Gamma_{k}\left(e+\frac{k}{2}\right) \Gamma_{k}(f) \Gamma_{k}\left(f+\frac{k}{2}\right)} \\
& \times G_{k, 3,4}^{4,1}\left[\begin{array}{c}
k, 2 e+2 f, \frac{k}{2}+e+f \\
2 e, 2 f, e+f, k
\end{array} \mid-p\right],
\end{aligned}
$$

where $e+f+\frac{k}{2}, e, f, e+\frac{k}{2}, f+\frac{k}{2} \in \mathbb{C} \backslash \mathbb{Z}_{0}^{-}, k>0$.

Case-11: By choosing $g=k, A=2, B=1, e_{1}=e, e_{2}=f+g, f_{1}=h, z=1, r=1$ in (2.1), we have

$$
\left\{{ }_{2} F_{1, k}\left[\begin{array}{c}
e, f+g \\
h
\end{array} \mid x\right]: p\right\}=\frac{\Gamma_{k}(h)}{p \Gamma_{k}(e) \Gamma_{k}(f+g)} G_{k, 2,3}^{3,1}\left[\begin{array}{c}
k, h \\
e, f+g, k
\end{array} \mid-p\right]
$$

and

$$
\left\{F_{1, k}(e ; f, g ; h ; x, x): p\right\}=\frac{\Gamma_{k}(h)}{p \Gamma_{k}(e) \Gamma_{k}(f+g)} G_{k, 2,3}^{3,1}\left[\begin{array}{c}
k, h \\
e, f+g, k
\end{array} \mid-p\right],
$$

where $h, e, f+g \in \mathbb{C} \backslash \mathbb{Z}_{0}^{-}, k>0$. 
Case-12: By choosing $g=k, A=1, B=1, e_{1}=e, f_{1}=f, z=1, r=1$ in (2.1), we have

$$
\left\{{ }_{1} F_{1, k}\left[\begin{array}{l}
e \\
f
\end{array} \mid x\right]: p\right\}=\frac{\Gamma_{k}(f)}{p \Gamma_{k}(e)} G_{k, 2,2}^{2,1}\left[\begin{array}{l}
k, f \\
e, k
\end{array} \mid-p\right]
$$

and

$$
\left\{e^{x}{ }_{1} F_{1, k}\left[\begin{array}{l}
e \\
f
\end{array} \mid-x\right]: p\right\}=\frac{\Gamma_{k}(f)}{p \Gamma_{k}(e)} G_{k, 2,2}^{2,1}\left[\begin{array}{l}
k, f \\
e, k
\end{array} \mid-p\right],
$$

where $e, f \in \mathbb{C} \backslash \mathbb{Z}_{0}^{-}, k>0$.

Case-13: By choosing $g=k, A=1, B=1, e_{1}=e, f_{1}=f, z=1, r=1$ in (2.1), we have

$$
\left\{{ }_{1} \mathrm{~F}_{1, \mathrm{k}}\left[\begin{array}{c|c}
\mathrm{e} \\
2 \mathrm{e}
\end{array} \mid 2 x\right]: \mathrm{p}\right\}=\frac{\Gamma_{\mathrm{k}}(2 e)}{\mathrm{p} \Gamma_{\mathrm{k}}(e)} \mathrm{G}_{\mathrm{k}, 2,2}^{2,1}\left[\begin{array}{c|c}
\mathrm{k}, 2 \mathrm{e} & -\mathrm{p} \\
\mathrm{e}, \mathrm{k} & \mathrm{2}
\end{array}\right] .
$$

By using Legendre's Duplication formula (1.1) for $k>0$, we have

$$
\left\{e^{x}{ }_{0} F_{1, k}\left[e+\frac{k}{2} \mid \frac{-x^{2}}{4}\right]: p\right\}=\frac{2^{\frac{2 e}{k}-1} \sqrt{k} \Gamma_{k}\left(e+\frac{k}{2}\right)}{p \sqrt{\pi}} G_{k, 2,2}^{2,1}\left[\begin{array}{c|c}
k, 2 e & -p \\
e, k & 2
\end{array}\right],
$$

where $e+\frac{k}{2} \in \mathbb{C} \backslash \mathbb{Z}_{0}^{-}, k>0$.

\section{Laplace transform of some other special functions}

In the this section, we find Laplace transform of some known special functions for different choices of parameters in (2.1) by using cancellation properties, Legendre's duplication and triplication formulas (1.1) and (1.2) for the product of gamma k-function and by applying reduction formulas in k-form, the classical version of which is given in [37].

Case-1: By choosing $g=v+q+k, A=2, B=3, e_{1}=\frac{v+q+k}{2}, e_{2}=\frac{v+q+2 k}{2}, f_{1}=v+k, f_{2}=q+k$, $f_{3}=v+q+k, z=-1, r=2$, in (2.1), we have

$$
\begin{aligned}
& \left\{x^{\frac{v+q+k}{k}-1}{ }_{2} F_{3, k}\left[\begin{array}{c}
\frac{v+q+k}{2}, \frac{v+q+2 k}{2} \\
v+k, q+k, v+q+k
\end{array} \mid-x^{2}\right]: p\right\} \\
& =\frac{\left(2 \frac{\pi}{k}\right)^{-\frac{1}{2}} \Gamma_{k}(v+k) \Gamma_{k}(q+k) \Gamma_{k}(v+q+k)(2)^{\frac{v+q+k}{k}-\frac{1}{2}}}{p^{\frac{v+q+k}{2}} \Gamma_{k}\left(\frac{v+q+k}{2}\right) \Gamma_{k}\left(\frac{v+q+2 k}{2}\right)} G_{k, 4,4}^{4,1}\left[\begin{array}{c}
k, v+k, q+k, v+q+k \\
\left.\frac{v+q+k}{2}, \frac{v+q+2 k}{2}, \frac{v+q+k}{2}, \frac{v+q+2 k}{2} \mid \frac{p^{2}}{4}\right] .
\end{array}\right.
\end{aligned}
$$

After some simplifications and using Theorem 2.4 we are at the result:

$$
\begin{aligned}
\left\{J_{v, k} J_{q, k}: p\right\} & =\frac{k 2^{\frac{v+q}{k}}}{\pi p^{\frac{v+q}{k}}+1} G_{k, 4,4}^{4,1}\left[\begin{array}{c}
k, v+k, q+k, v+q+k \\
\frac{v+\mathrm{q}+k}{2}, \frac{v+q+2 k}{2}, \frac{v+q+k}{2}, \frac{v+q+2 k}{2} \mid \frac{p^{2}}{4}
\end{array}\right] \\
& =\frac{\Gamma_{k}(v+q+k)}{2^{\frac{v+q}{k}} p^{\frac{v+q}{k}+1} \Gamma_{k}(v+k) \Gamma_{k}(q+k)}{ }_{4} F_{3, k}\left[\frac{\frac{v+q+k}{2}, \frac{v+q+2 k}{2}}{v+k+q+k}, \frac{v+q+2 k}{2} \mid \frac{-4}{p^{2}}\right],
\end{aligned}
$$

where $J_{v, k}$ and $J_{q, k}$ are the Bessel $k$-functions, $v+q+k, q+k, v+k \in \mathbb{C} \backslash \mathbb{Z}_{0}^{-}$.

Case-2: By choosing $g=2 q+2 k, A=1, B=2, e_{1}=q+\frac{3 k}{2}, f_{1}=q+2 k, f_{2}=2 q+2 k, z=-1, r=2$, in (2.1), we have

$$
\left\{x^{\frac{2 q+2 k}{k}-1}{ }_{1} F_{2, k}\left[\begin{array}{c}
q+\frac{3 k}{2} \\
q+2 k, 2 q+2 k
\end{array} \mid-x^{2}\right] ; p\right\}=\frac{\left(2 \frac{\pi}{k}\right)^{-\frac{1}{2}} \Gamma_{k}(q+2 k) \Gamma_{k}(2 q+2 k)(2)^{\frac{2 q}{k}+\frac{1}{2}}}{p^{\frac{2 q}{k}+2} \Gamma_{k}\left(q+\frac{3 k}{2}\right)}
$$




$$
\times G_{k, 3,3}^{3,1}\left[\begin{array}{c}
k, q+k, 2 q+2 k \\
q+\frac{3 k}{2}, q+k, q+\frac{3 k}{2} \mid \frac{p^{2}}{4}
\end{array}\right]
$$

which yields:

$$
\begin{aligned}
& \left\{J_{q, k} J_{q+1, k} ; p\right\}=\frac{k 2^{\frac{2 q}{k}+1}}{\pi p^{\frac{2 q}{k}}+2} G_{k, 3,3}^{3,1}\left[\begin{array}{c}
k, q+2 k, 2 q+2 k \\
q+\frac{3 k}{2}, q+k, q+\frac{3 k}{2}
\end{array} \mid \frac{p^{2}}{4}\right] \\
& =\frac{\sqrt{k} \Gamma_{k}\left(q+\frac{3 k}{2}\right)}{\sqrt{\pi} p^{\frac{2 q}{k}+2} \Gamma_{k}(q+2 k)}{ }_{3} F_{2, k}\left[\begin{array}{c}
q+k, q+\frac{3 k}{2}, q+\frac{3 k}{2} \mid \frac{-4}{p^{2}} \\
q+2 k, 2 q+2 k
\end{array}\right. \text {, }
\end{aligned}
$$

where $J_{q, k}$ and $J_{q+1, k}$ are the Bessel $k$-functions $q+\frac{3 k}{2}, q+2 k \in \mathbb{C} \backslash \mathbb{Z}_{0}^{-}$.

Case-3: By choosing $g=2 q+k, A=1, B=2, e_{1}=q+\frac{k}{2}, f_{1}=q+k, f_{2}=2 q+k, z=-1, r=2$, in (2.1), we obtain the following result:

$$
\begin{aligned}
\left\{x^{\frac{2 q+k}{k}-1}{ }_{1} F_{2, k}\left[\begin{array}{c}
q+\frac{k}{2} \\
q+k, 2 q+k
\end{array} \mid-x^{2}\right] ; p\right\} & =\frac{\sqrt{k} \Gamma_{k}(q+k) \Gamma_{k}(2 q+k)(2)^{\frac{2 q}{k}}}{\sqrt{\pi} p^{\frac{2 q}{k}}+1 \Gamma_{k}\left(q+\frac{k}{2}\right)} G_{k, 2,2}^{2,1}\left[\begin{array}{c}
k, 2 q+k \\
q+\frac{k}{2}, q+\frac{k}{2}
\end{array} \mid \frac{p^{2}}{4}\right], \\
\left\{J_{q}^{2}(x) ; p\right\} & =\frac{k(2)^{\frac{2 q}{k}}}{\pi p^{\frac{2 q}{k}}+1} G_{k, 2,2}^{2,1}\left[\begin{array}{c}
k, 2 q+k \\
q+\frac{k}{2}, q+\frac{k}{2}
\end{array} \mid \frac{p^{2}}{4}\right] \\
& =\frac{\sqrt{k} \Gamma_{k}\left(q+\frac{k}{2}\right)}{\sqrt{\pi} p^{\frac{2 q}{k}+1} \Gamma_{k}(q+k)}{ }_{2} F_{1, k}\left[\begin{array}{c}
q+\frac{k}{2}, q+\frac{k}{2} \mid \\
2 q+k
\end{array} \mid \frac{-4}{p^{2}}\right] .
\end{aligned}
$$

Case-4: By choosing $g=k, A=1, B=2, e_{1}=\frac{k}{2}, f_{1}=q+k, f_{2}=q+k, z=-1, r=2$, in (2.1), we have

$$
\left\{x^{\frac{k}{k}-1}{ }_{1} F_{2, k}\left[\begin{array}{c}
\frac{k}{2} \\
q+k, q+k
\end{array} \mid-x^{2}\right] ; p\right\}=\frac{k \Gamma_{k}(-q+k) \Gamma_{k}(q+k)}{\pi p} G_{k, 3,3}^{3,1}\left[\begin{array}{c}
k,-q+k, q+k \mid \frac{p^{2}}{4} \\
\frac{k}{2}, \frac{k}{2}, k
\end{array}\right] .
$$

By using Theorem 2.5 we have

$$
\left\{J_{q, k}(x) J_{-q, k}(x) ; p\right\}=\frac{k}{\pi p} G_{k, 3,3}^{3,1}\left[\begin{array}{c|c}
k,-q+k, q+k & p^{2} \\
\frac{k}{2}, \frac{k}{2}, k
\end{array}\right]=\frac{\sin \frac{\pi q}{k}}{\pi p q}{ }_{3} F_{2, k}\left[\begin{array}{c}
\frac{k}{2}, \frac{k}{2}, k \\
-q+k, q+k
\end{array} \mid \frac{-4}{p^{2}}\right] .
$$

Case-5: By choosing $g=2 q+k, A=0, B=3, f_{1}=\frac{q+k}{2}, f_{2}=\frac{q+2 k}{2}, f_{3}=q+k, z=\frac{-1}{64}, r=4$, in (2.1), we have

$$
\begin{aligned}
& \left\{\frac{x^{\frac{2 q}{k}}}{2^{\frac{2 q}{k}}} 0_{3, k}\left[\frac{q+k}{2}, \frac{q+2 k}{2}, q+k \mid \frac{-x^{4}}{64}\right]\right\}=\frac{k \Gamma_{k}(q+k) \Gamma_{k}(q+k)(2)^{\frac{2 q}{k}}-\frac{1}{2}}{\pi\left(2 \frac{2 q}{k} p^{\frac{2 q}{k}+1}\right.} G_{k, 2,2}^{2,1}\left[\frac{2 q+k}{4}, \frac{2 q+3 k}{4} \mid \frac{p^{4}}{4}\right],
\end{aligned}
$$

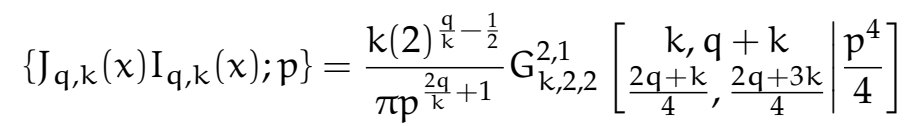

$$
\begin{aligned}
& =\frac{\sqrt{k} \Gamma_{k}\left(\frac{2 q+k}{2}\right)}{\sqrt{\pi} p^{\frac{2 q}{k}+1} \Gamma_{k}(q+k)}{ }_{2} F_{1, k}\left[\frac{2 q+k}{4}, \frac{2 q+3 k}{4} \mid \frac{-4}{p^{4}}\right] \text {. }
\end{aligned}
$$

The Classical form of products $J_{v, k} J_{q, k}, J_{q, k} J_{q+1, k}, J_{q, k}^{2}, J_{q, k} J_{-q, k}, J_{q, k}(x) I_{q, k}$ is given in [14].

Case-6: By choosing $g=l+h+k, A=0, B=2, f_{1}=l+k, f_{2}=h+k, z=\frac{-1}{27}, r=3$, in (2.1), we have

$$
\left\{x^{\frac{l+h}{k}}{ }_{0} F_{2, k}\left[\begin{array}{c}
- \\
l+k, h+k
\end{array} \mid \frac{-x^{3}}{27}\right] ; p\right\}=\frac{k \Gamma_{k}(l+k) \Gamma_{k}(h+k)(3)^{\frac{l+h}{k}+\frac{1}{2}}}{2 \pi p^{\frac{l+h}{k}+1}} G_{k, 3,3}^{3,1}\left[\begin{array}{c}
k, l+k, h+k \\
\frac{l+h+k}{3}, \frac{l+h+2 k}{3}, \frac{l+h+3 k}{3} \mid
\end{array} \mid p^{3}\right],
$$




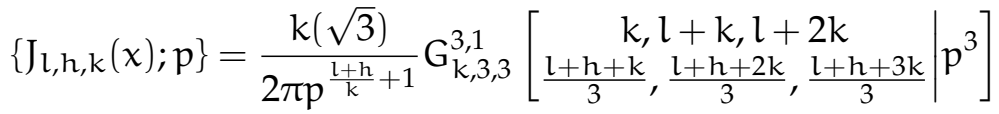

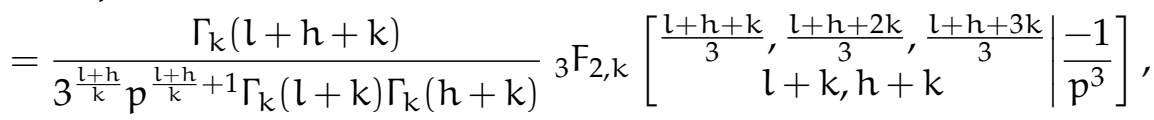

where $J_{l, h, k}$ is the Hyper-Bessel $k$-function of Humbert, the classical form of which is given in [10].

Case-7: By choosing $g=e+k, A=1, B=1, e_{1}=e f_{1}=e+k, z=-1, r=1$, in (2.1), we have

$$
\left\{x^{\frac{e}{k}}{ }_{1} F_{1, k}\left[\begin{array}{c}
e \\
e+k
\end{array} \mid-x\right] ; p\right\}=\frac{\Gamma_{k}(e+k)}{p^{\frac{e}{k}+1} \Gamma_{k}(e)} G_{k, 1,1}^{1,1}\left[\begin{array}{c}
k \\
e
\end{array} \mid p\right] \text {. }
$$

where $\gamma_{\mathrm{k}}$ is incomplete gamma function $e \in \mathbb{C} \backslash \mathbb{Z}_{0}^{-}$.

Case-8: By choosing $g=2 k, A=1, B=2, e_{1}=\frac{k}{2}, f_{1}=\frac{3 k}{2}, f_{2}=\frac{3 k}{2}, z=\frac{-1}{4}, r=2$, in (2.1), we have

$$
\left\{x_{1} F_{2, k}\left[\frac{\frac{k}{2}}{\frac{3 k}{2}}, \frac{3 k}{2} \mid-\frac{x^{2}}{4}\right] ; p\right\}=\frac{k^{2}}{2 p^{2}} G_{k, 2,2}^{2,1}\left[\begin{array}{c}
k, \frac{3 k}{2} \\
\frac{k}{2}, k
\end{array} \mid p^{2}\right]
$$

and

$$
\left\{M_{i, k}(x) ; p\right\}=\frac{k}{p^{2}}{ }_{2} F_{1, k}\left[\begin{array}{c}
\frac{k}{2}, k \\
\frac{3 k}{2}
\end{array} \mid-\frac{1}{p^{2}}\right],
$$

where $M_{i, k}(x)$ is the sine $k$-integral.

Case-9: By choosing $g=2 k, A=1, B=2, e_{1}=\frac{k}{2}, f_{1}=\frac{3 k}{2}, f_{2}=\frac{3 k}{2}, z=\frac{1}{4}, r=2$, in (2.1), we have

$$
\left\{x_{1} F_{2, k}\left[\frac{\frac{k}{2}}{2}, \frac{3 k}{2} \mid \frac{x^{2}}{4}\right] ; p\right\}=\frac{k^{2}}{2 p^{2}} G_{k, 2,2}^{2,1}\left[\begin{array}{c}
k, \frac{3 k}{2} \\
\frac{k}{2}, k
\end{array} \mid-p^{2}\right]
$$

and

$$
\left\{M_{h i, k}(x) ; p\right\}=\frac{k}{p^{2}}{ }_{2} F_{1, k}\left[\begin{array}{c|c}
\frac{k}{2}, k \\
\frac{3 k}{2} & \frac{1}{p^{2}}
\end{array},\right.
$$

where $M_{h i, k}(x)$ is the sine hyperbolic k-integral.

Case-10: By choosing $g=k, A=0, B=3, f_{1}=\frac{k}{2}, f_{2}=\frac{k}{2}, f_{3}=k, z=-\frac{1}{256}, r=4$, in (2.1), we have

$$
\left\{{ }_{0} \mathrm{~F}_{3, \mathrm{k}}\left[\underset{\frac{\mathrm{k}}{2}, \frac{\mathrm{k}}{2}, \mathrm{k}}{ } \mid-\frac{x^{4}}{256}\right] ; \mathrm{p}\right\}=\frac{\sqrt{\mathrm{k}}}{\sqrt{2 \pi} \mathrm{p}} \mathrm{G}_{\mathrm{k}, 2,2}^{2,1}\left[\begin{array}{c}
\mathrm{k}, \frac{\mathrm{k}}{2} \\
\frac{\mathrm{k}}{4}, \frac{3 \mathrm{k}}{4}
\end{array} \mid \mathrm{p}^{4}\right]
$$

and

$$
\{\operatorname{ber}(x, k) ; p\}=\frac{1}{p}{ }_{2} F_{1, k}\left[\begin{array}{c}
\frac{k}{4}, \frac{3 k}{4} \\
\frac{k}{2}
\end{array} \mid-\frac{1}{p^{4}}\right],
$$

where ber $(x, k)$ is the Kelvin's k-function.

The Sine integral, Sine hyperbolic integral, and Kelvin's function are given in [12].

\section{Conclusions}

In this research work we gave the definitions of generalized Meijer G-function and k-Laplace transform and proved some important results. We then find Laplace transform of hypergeometric $k$-functions and some other special $k$-functions, $k>0$ in the form of generalized Meijer $G$-functions that is Meijer $(G, k)$ functions. The results obtained in this paper are more general, which can be reduced to classical version by taking $k \rightarrow 1$. 


\section{References}

[1] V. Adamchik, The evaluation of integrals of Bessel functions via G-function identities, J. Comput. Appl. Math., 64 (1995), 283-290. 1

[2] G. Agarwala, A. Gaura, K. S. Nisarb, A. H. Abusufianb, On some properties of $\beta$-Laplace integral transform, J. Math. Computer Sci., 23 (2021), 315-320. 1

[3] R. Beals, J. Szmigielski, Meijer G-function: A Gentle Introduction, Notices Amer. Math. Soc., 60 (2013), 866-872. 1

[4] J. S. Choi, P. Agarwal, Certain unified integrals associated with Bessel functions, Bound. Value Probl., 2013 (2013), 9 pages. 1.2

[5] R. Diaz, E. Pariguan, On hypergeometric functions and pochhammer k-symbol, Divulg. Mat., 15 (2007), 179-192. 1, 1.5

[6] R. Diaz, C. Teruel, q, k-Generalized Gamma and Beta Functions, J. Nonlinear Math. Phys., 12 (2008), 118-134. 1

[7] G. Doetsch, Introduction to the theory and applications of the Laplace transformation, Springer-Verlag, New York, (1970). 1

[8] A. Erdélyi, W. Magnus, F. Oberhettinger, F. G. Tricomi, Higher Transcendental Functions, Vol. I, McGraw-Hill Book Co., New York-Toronto-London, (1953). 1

[9] A. Erdélyi, W. Magnus, F. Oberhettinger, F. G. Tricomi, Tables of Integral Transforms, Vol. I, McGraw-Hill Book Co., New York-Toronto-London, (1954). 1

[10] A. Erdélyi, W. Magnus, F. Oberhettinger, F. G. Tricomi, Higher Transcendental Function, Vol. III, McGraw-Hill Book Company, New York-Toronto-London, (1955). 4

[11] R. Garra, R. Goreno, F. Polito, Tomovski, Z. Tomovski, Hilfer-Prabhakar derivatives and some applications, Appl. Math. Comput., 242 (2014), 576-589. 1.2

[12] I. S. Gradshteyn, I. M. Ryzhik, Table of integrals, series, and products, Academic Press, San Diego, (2007). 4

[13] C. G. Kokologiannaki, Properties and inequalities of generalized k-gamma, beta and zeta functions, Int. J. Contemp. Math. Sci., 5 (2010), 653-660. 1

[14] Y. L. Luke, The Special Functions and Their Approximations, Academic Press, New York-London, (1969). 1, 1, 4

[15] M. Mansour, Determining the k-generalized gamma function $\Gamma_{\mathrm{k}}(\mathrm{x})$ by functional equations, Int. J. Contemp. Math. Sci., 4 (2009), 1037-1042. 1

[16] A. M. Mathai, R. K. Saxena, Generalized Hypergeometric Functions with Applications in Statistics and Physical Sciences, Springer-Verlag, New York, (1973). 1

[17] A. M. Mathai, R. K. Saxena, H. J. Haubold, The H-Function, Springer, New York, (2010). 1

[18] C. S. Meijer, Über Whittakersche Bezw. Besselsche Funktionen und deren Produkte, Nieuw. Arch. Wiskunde, 18 (1936), 10-39. 1

[19] C. S. Meijer, Multiplikationstheoreme für die Funktion $\mathrm{G}_{\mathrm{p}, \mathrm{q}}^{\mathrm{m}, \mathrm{n}}(z)$, Nederl. Akad. Wetensch. Proc. Ser. A, 44 (1941), 1062-1070. 1

[20] C. S. Meijer, Neue Integralstellungen für Whittakersche Funktionen I, Nederl. Akad. Wetensch. Proc. Ser. A, 44 (1941), 81-92. 1

[21] C. S. Meijer, Expension theorems for the G-function, V. Nederl. Akad. Wetensch. Proc. Ser. A. $56=$ Indagationes Math., 15 (1953), 349-397. 1

[22] S. Mubeen, G. M. Habibullah, k-fractional integrals and application, Int. J. Contemp. Math. Sci., 7 (2012), 89-94. 1

[23] S. Mubeen, S. D. Purohit, M. Arshad, G. Rahman, Extension of k-gamma, k-beta functions and k-beta distribution, J. Math. Anal., 7 (2016), 118-131. 1

[24] K. S. Nisar, S. R. Mondal, J. S. Choi, Certain inequalities involving the k-Struve function, J. Inequal. Appl., 2017 (2017), 8 pages. 1

[25] K. S. Nisar, G. Rahman, J. S. Choi, S. Mubeen, M. Arshad, Generalized hypergeometric k-functions via (k, s)-fractional calculus, J. Nonlinear Sci. Appl., 10 (2017), 1791-1800. 1

[26] K. S. Nisar, G. Rahman, J. S. Choi, S. Mubeen, M. Arshad, Certain Gronwall type inequalities associated with RiemannLiouville k-and hadamard k-fractional derivatives and their applications, East Asian Math. J., 34 (2018), 249-263. 1

[27] F. E. Nixon, Handbook of Laplace Transformation, Prentice-Hall, Englewood Cliffs, (1960). 1

[28] N. E. Norlund, Hypergeometric Functions, Acta Math., 94 (1955), 289-349. 1

[29] F. Oberhettinger, L. Badii, Tables of Laplace Transforms, Springer-Verlag, New York-Heidelberg, (1973). 1

[30] A. Pishkoo, A G-function form of radial states in carbon and hydrogen atoms: absorption or emission of radiation, Adv. Stud. Theor. Phys., 9 (2015), 145-154. 1

[31] A. P. Prudnikov, Y. A. Brychkov, O. I. Marichev, Integrals and Series, Vol. 3, Gordon and Breach Science Publishers, New York, (1990). 1

[32] A. P. Prudnikov, Y. A. Brychkov, O. I. Marichev, Integrals and Series, Vol. 4, Gordon and Breach Science Publishers, New York, (1992). 1

[33] A. P. Prudnikov, Y. A. Brychkov, O. I. Marichev, Integrals and Series, Vol. 5, Gordon and Breach Science Publishers, New York, (1992). 1

[34] M. I. Qureshi, D. K. Kabra, M. S. Baboo, Laplace Transforms of Some Special Functions of Mathematical Physics Using Mellin-Barnes Type Contour Integration, Int. J. Math. Stat. Invent., 3 (2015), 21-34. 1, 2

[35] G. Rahman, K. S. Nisar, T. Kim, S. Mubeen, M. Arshad, The Extended k-Mittag-Leffler Function and Its Properties, Proc. Jangjeon Math. Soc., 21 (2018), 487-495. 1 
[36] G. Rahman, K. S. Nisar, T. Kim, S. Mubeen, M. Arshad, Inequalities Involving Extended k-Gamma and k-Beta Functions, Proc. Jangjeon Math. Soc., 21 (2018), 143-153. 1

[37] E. D. Rainville, Special functions, Macmillan Co., New York, (1960). 1.1, 1.4, 1.7, 3,4

[38] M. Saif, F. Khan, K. S. Nisar, S. Araci, Modified Laplace transform and its properties, J. Math. Computer Sci., 21 (2020), 127-135. 1

[39] L. J. Slater, Generalized Hypergeometric Functions, Cambridge University Press, Cambridge, (1966). 1 